\title{
Polymeric nanoparticles improved Curcumin brain delivery and its therapeutic efficacy against intracerebral hemorrhage
}

\section{Cong Yang}

Guangzhou University of Chinese Medicine

\section{Meng-Meng Han}

Guangzhou University of Chinese Medicine

\section{Ruo-Yu Li}

Guangzhou University of Chinese Medicine

\section{Yu-Na Yang}

Guangzhou University of Chinese Medicine

\section{Li-Gui Zhou}

Guangzhou University of Chinese Medicine

\section{Li-Ning Duan}

Guangzhou University of Chinese Medicine

\section{Shi-Yu Su}

Guangzhou University of Chinese Medicine

\section{Min Li}

Guangzhou University of Chinese Medicine

\section{Qi Wang}

Guangzhou University of Chinese Medicine

\section{Tong-Kai Chen}

Guangzhou University of Chinese Medicine

\section{Yousheng Mo ( $\nabla 378480308 @ q q . c o m$ )}

Guangdong Provincial Hospital of Traditional Chinese Medicine https://orcid.org/0000-0001-75996015

\section{Research Article}

Keywords: Polymer Nanoparticles, Curcumin, Brain delivery, Ferroptosis

Posted Date: July 19th, 2021

DOI: https://doi.org/10.21203/rs.3.rs-713424/v1 
License: (c) (i) This work is licensed under a Creative Commons Attribution 4.0 International License. Read Full License 


\section{Polymeric nanoparticles improved Curcumin brain delivery and its therapeutic efficacy against intracerebral hemorrhage}

Cong Yang ${ }^{1^{\dagger}}$, Meng-Meng Han ${ }^{1,2}{ }^{\dagger}$, Ruo-Yu Li ${ }^{1}{ }^{\dagger}$, Yu-Na Yang ${ }^{1,3}$, Li-Gui Zhou ${ }^{1,3}$, Li-Ning Duan ${ }^{1,4}$, Shi-Yu Su ${ }^{1,4}$, Min $\mathrm{Li}^{1,4}$, Qi Wang ${ }^{1 *}$, Tong-Kai Chen ${ }^{1 *}$, You-Sheng $\mathrm{Mo}^{1,5^{*}}$

*Correspondence: wangqi@gzucm.edu.cn; chentongkai@gzucm.edu.cn; 378480308@qq.com

Cong Yang, Meng-Meng Han and Ruo-Yu Li contributed equally to this work.

${ }^{1}$ Science and Technology Innovation Center, Guangzhou University of Chinese Medicine, Guangzhou 510405, China

${ }^{2}$ The First Affiliated Hospital of Guangzhou University of Chinese Medicine, Guangzhou 510405, China.

${ }^{3}$ Laboratory Animal Center, Guangzhou University of Chinese Medicine, Guangzhou 510405, China

${ }^{4}$ Clinical Medical College of Acupuncture Moxibustion and Rehabilitation, Guangzhou University of Chinese Medicine, Guangzhou 510405, China

${ }^{5}$ The Second Affiliated Hospital of Guangzhou University of Chinese Medicine, Guangzhou 510120, China.

Full list of author information is available at the end of the article.

\section{Abstract}


Background: Intracerebral hemorrhage (ICH) is a severe type of stroke. Ferroptosis is a new form of regulated cell death, which plays an indispensable role in the pathological process of $\mathrm{ICH}$. Curcumin (Cur), a widespread phenolic compound, is derived from the rhizome of Curcuma longa. It could attenuate hematoma volume and neurological injury in ICH. Nevertheless, its poor solubility in water, low oral bioavailability and difficulty in transporting across physiological barriers led to poor efficacy. Polymer nanoparticles (NPs) are widely used drug delivery matrix material with good biocompatibility, which are reported to improve the bioavailability and pharmacokinetic profiles of drugs. In this study, we utilized NPs to encapsulate Cur and analyzed the effect of Cur-NPs on Cur brain delivery and its therapeutic efficacy against ICH.

Results: The spherical Cur-NPs had a particle size $127.31 \pm 2.73 \mathrm{~nm}$, a PDI of 0.21 \pm 0.01 and a zeta potential of $-0.25 \pm 0.02 \mathrm{mV}$. Cur-NPs could draw into Madin-Darby canine kidney (MDCK) cells through a number of nonspecific endocytosis mechanisms, mainly mediated via clathrin and plasma membrane microcapsules. Moreover, Cur-NPs tended to accumulate in the endoplasmic reticulum and lysosome. In a zebrafish model, Cur-NPs could transport across physiological barriers. In a C57BL/6 mice model, we found that Cur-NPs had more desirable improvements in Cur accumulation within the plasma and brain. Importantly, in an ICH mouse model, we confirmed that Cur-NPs were an effective treatment for ICH. Finally, Cur-NPs effectively inhibited ferroptosis caused by erastin in HT22 mouse hippocampal cells. 
Conclusion: Cur-NPs represent a potentially effective strategy to enhance Cur brain delivery and therapeutic efficacy in the treatment of $\mathrm{ICH}$.

Keywords: Polymer Nanoparticles, Curcumin, Brain delivery, Ferroptosis

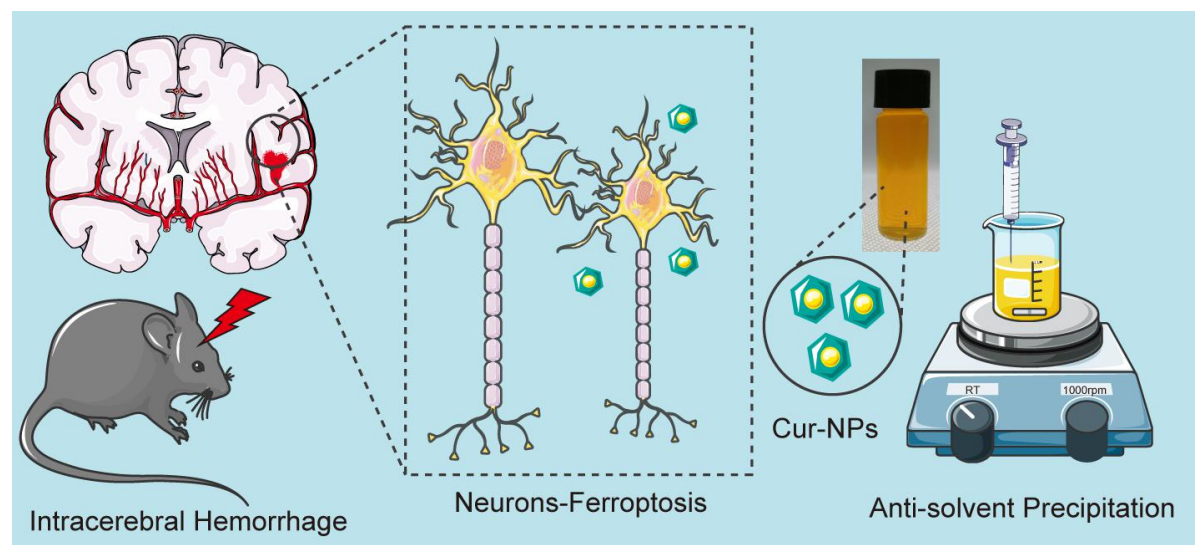

Scheme 1 Polymer Nanoparticles (NPs) increase Curcumin (Cur) oral bioavailability in intracerebral hemorrhage (ICH) and the anti-ferroptosis machanism of Cur-NPs.

\section{Background}

Intracerebral hemorrhage ( $\mathrm{ICH})$ is a severe type of stroke, which leads to $15-25 \%$ of strokes[1]. The survival rate of 1 -year in ICH patients is less than $40 \%$ [2]. The specific mechanism of severe neurological injury occurring following ICH is not clear. During the acute phase of ICH, hemoglobin is quickly degraded into iron, carbon monoxide and biliverdin. Large amounts of iron are thus released into the extracellular space[3]. Accumulating evidence suggested that iron overload could be a potent contributor to perihematomal edema, peroxide accumulation and cell death[4]. Ferroptosis is a new form of regulated cell death triggered by lipid peroxidation in an iron-dependent manner [5]. Resent studies demonstrated that ferroptosis plays an indispensable role in the pathological process of $\mathrm{ICH}[6]$. Targeting ferroptosis may represent a novel therapeutic strategy for the treatment of 
$\mathrm{ICH}[7]$.

Although a degree of efficacy can be achieved through the surgical evacuation of hematoma in ICH patients, the clinical applications of surgery is limited by its high costs and significant risks[8-10]. Unfortunately, effective pharmaceutical approaches for ICH are lacking at present[11]. One of the challenges in drug development is the poor oral bioavailability and brain accumulation[12, 13].To resolve these puzzles, extensive research efforts have been pursued in the quest for novel compounds from herbal medicines[14].

Curcumin (Cur), a widespread phenolic compound, is derived from the rhizome of Curcuma longa. Recent studies have reported that Cur has widely pharmacological functions including anti-oxidation, anti-inflammation and neuroprotective effects[15-17]. Crucially, Cur effectively attenuated the hematoma volume and neurological injury in ICH model mice[18]. Nevertheless, its poor solubility in water, low oral bioavailability and difficulty in transporting across physiological barriers reduce the efficacy[19]. Multiple efforts have been devoted to improve Cur performance via encapsulating Cur in liposomes, polymeric micelles, microspheres and solid lipid nanoparticles[20-22]. These strategies increased Cur bioavailability to some degrees, but no studies showed the pharmacokinetics of drugs and whether they can cross biological barriers remains not elucidated. So, it is urgent to develop a new strategy which can increase the oral bioavailability and brain accumulation of Cur in ICH treatment .

Polymer Nanoparticles (NPs) are extensively used drug delivery matrix material 
with good biocompatibility, which have been approved by the US Food and Drug Administration[23, 24]. Previous work reported that NPs can improve the efficacy, solubility, bioavailability and pharmacokinetic profiles of drugs[25, 26]. Importantly, NPs have many advantages over other delivery platforms with multifunctionality, less toxicity and lower immune response[27]. Furthermore, NPs are prepared by an anti-solvent precipitation method, which is cheap and easy to perform[28, 29].

In this work, we utilized an anti-solvent precipitation to make Cur polymer nanoparticles (Cur-NPs). Madin Darby canine kidney (MDCK) cells have tight junctions and polarized mucus layersare, which are similar to intestinal epithelial cells[30]. So we employed them as an in vitro drug absorption model. The endothelial tight junction-based blood-brain barrier $(\mathrm{BBB})$ in zebrafish is similar to that of higher vertebrates[31]. Therefore, we used zebrafish to investigate the in vivo distribution and elimination process of Cur-NPs. Furthermore, we analyzed the plasma and brain pharmacokinetics of Cur-NPs in mice and assessed their neuroprotective effects on ICH model mice. Finally, a vitro study was conducted to evaluate the anti-ferroptosis effect of Cur-NPs on erastin-induced HT22 mouse hippocampal cells.

\section{Results and Discussion}

\section{Cur-NPs characterization}

In this study, Cur-NPs were made by an anti-solvent precipitation method (Fig.1A). Cur-NPs were predominantly spherical and ranged from $70 \mathrm{~nm}$ to $100 \mathrm{~nm}$ in diameter under transmission electron microscope (TEM) (Fig. 1B). The dynamic light scattering (DLS) detection showed a particle size of $127.31 \pm 2.73 \mathrm{~nm}$, a PDI of 
$0.21 \pm 0.01$ (Fig. 1C) and a zeta potential of $-0.25 \pm 0.02 \mathrm{mV}$ (Fig. 1D). The results of powder X-ray diffraction (XRD) showed that there were no distinctive Cur peaks at $17^{\circ}$ in Cur-NPs, potentially due to the larger Cur-NPs percentage resulting in masking of Cur peaks (Fig. 1E).

A

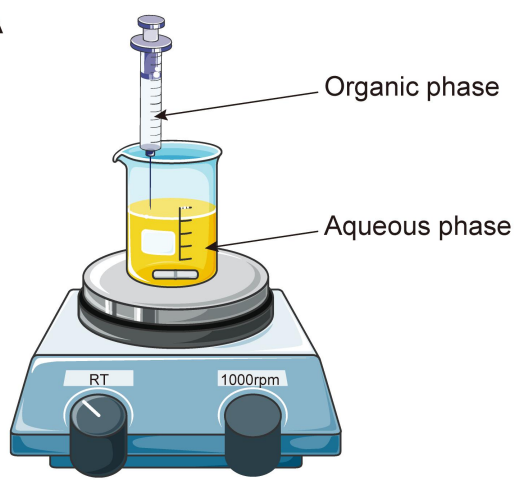

C

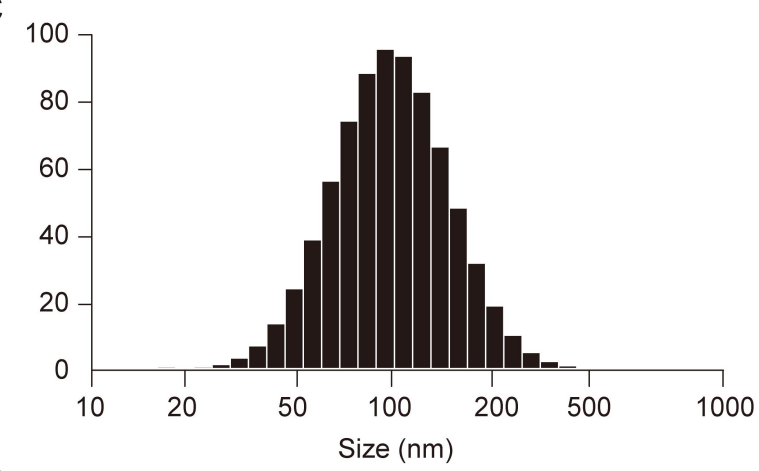

B
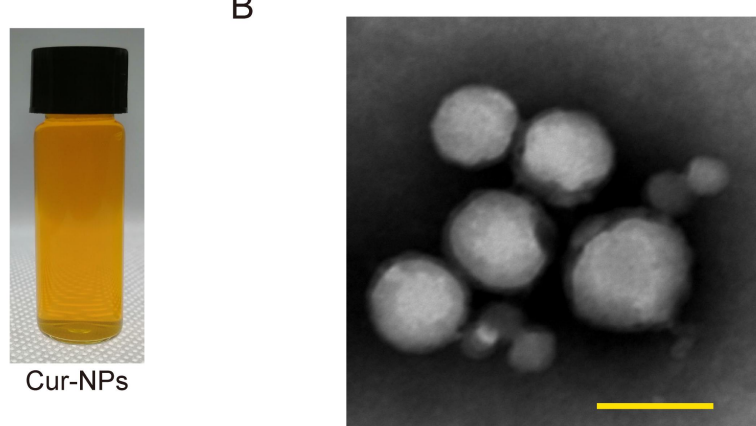

D

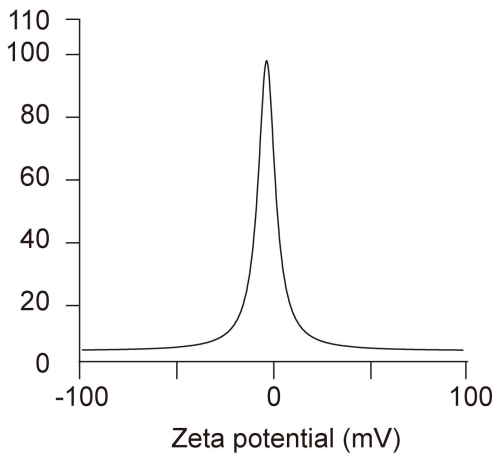

E

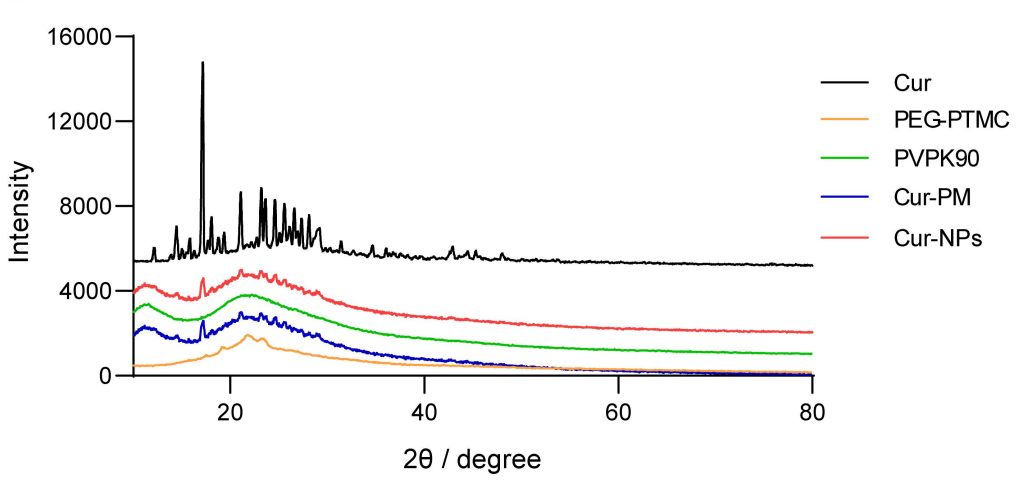

Fig. 1 Cur-NPs characterization. A Cur-NPs preparation. B TEM image. Scale bar: $100 \mathrm{~nm}$. C

Size distribution. D Zeta potential. E XRD of Cur, PEG-PTMC, PVPK90, Cur-PM (physical mixtures of HPMC E50 and Cur) and Cur-NPs. 


\section{Cur-NPs cellular uptake and distribution assessment}

The cellular uptake progress of Cur-NPs into the MDCK cells was in a time-dependent and concentration-dependent manner (Fig. 2A). To determine the cellular uptake mechanism of Cur-NPs in vitro, MDCK cells were incubated with different kinds of endocytosis inhibitors. Among these inhibitors, Chlorpromazine (CPZ), Hypertonic sucrose (HS) and Methyl- $\beta$-cyclodextrin (M $\beta C D)$ most obviously suppressed the endocytosis of Cur-NPs. These findings strongly indicated that Cur-NPs could be internalized through multiple endocytic nonspecifical mechanisms

(Fig. 2B). Next, we explored the cellular distribution of Cur-NPs in vitro. In this work, Cur-NPs were mainly distributed in endoplasmic reticulum (ER) and lysosomes (Lyso), which can regulate the drug bioavailability (Fig. 2C). Importantly, NPs showed negligible colocalization with mitochondria (Mito), which indicated that Cur-NPs existed no cytotoxicity in the mitochondria-dependent pathway[32] (Fig. 2D). 
A

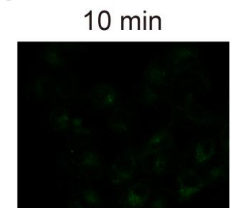

$0.5 \mu \mathrm{g} / \mathrm{ml}$

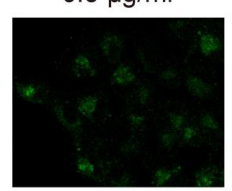

$\mathrm{C}$

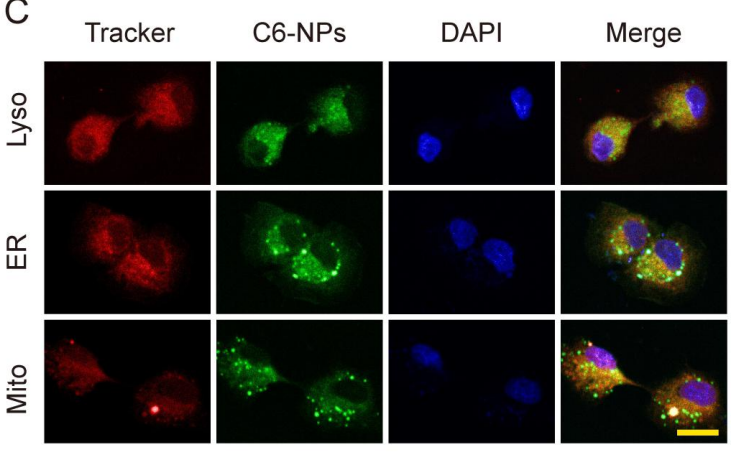

B
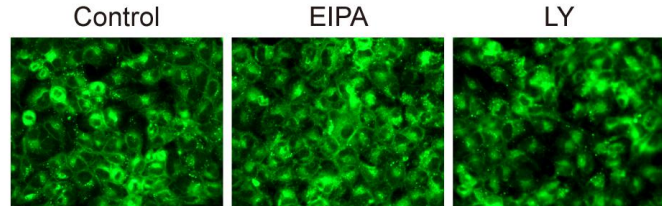

CPZ

HS
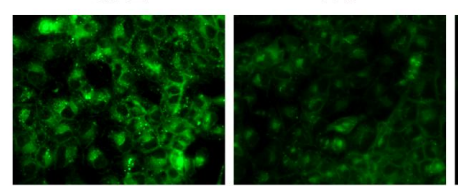

$M \beta C D$

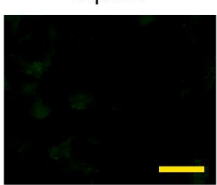

D

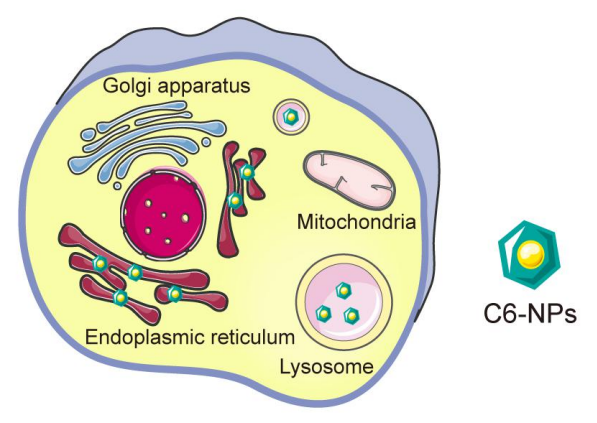

Fig. 2 Cur-NPs cellular uptake and distribution assessment. A The uptake of Cur-NPs was time-dependent and concentration-dependent. Scale bar: $50 \mu \mathrm{m}$. B The fluorescence images of endocytosis governing Cur-NPs uptake into MDCK cells. Scale bar: $50 \mu \mathrm{m}$. C The distribution of C6/Cur-NPs on organelles in cells. Scale bar: $25 \mu \mathrm{m}$. D The sketch map of cellular uptake process of C6/Cur-NPs.

\section{Analysis of Cur-NPs biodistribution in vivo}

To determine the in vivo biodistribution of Cur-NPs after oral administration, we incubated zebrafish with free C6 or C6/Cur-NPs. In the C6/Cur-NPs group, from 15 min to $60 \mathrm{~min}$, the fluorescence in the intestine and brain was significantly enhanced in a time-dependent manner. In contrast, the free C6 group showed minimal fluorescence. These results clearly suggested the higher absorption of the fluorescence delivered as the NPs, which strongly indicated that the NPs can cross BBB and lead to 
superior brain accumulation. Interestingly, C6/Cur-NPs were distributed to the eyes, which indicated that NPs can also cross blood retinal barrier. On the basis of these results, NPs were able to improve the absorption and brain accumulation of Cur (Fig. 3A). The concentration of Cur-NPs in the plasma of C57BL/6 mice was significantly higher than that received Cur alone (Fig. 3B). These findings provided strong evidence that NPs loading of Cur could significantly increase the systemic circulation because of the advantageous particle size and surface properties of NPs. The concentration of Cur-NPs in the brains was remarkably higher than that of the control group, reaching a peak at $8 \mathrm{~h}$, which indicated that the prolonged sustained release of Cur-NPs in the brain lead to better brain accumulation (Fig. 3C). Moreover, the concentration of Cur-NPs in heart, liver, spleen, lung and kidney were also significantly higher than those in the control, which may be related to the prolonged sustained release of Cur-NPs and its high plasma exposure rate (Fig. 3D-H) [33]. 

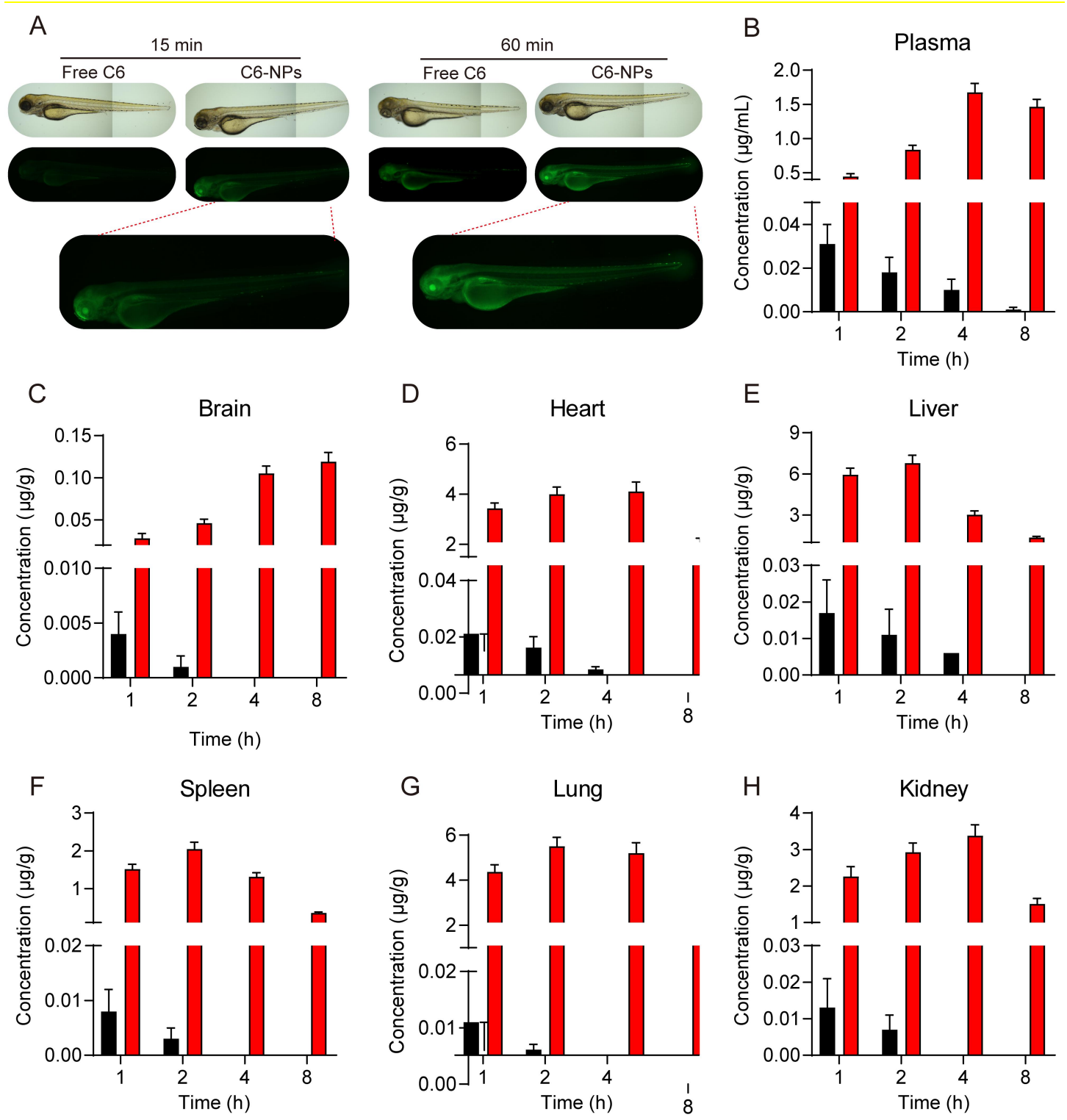

Fig. 3 A Analysis of Cur-NPs biodistribution in vivo. Zebrafish larvae at $7 \mathrm{dpf}$ following C6/Cur-NPs treatments $(400 \mathrm{ng} / \mathrm{mL})$ for 15 and $60 \mathrm{~min}$. Scale bar: $100 \mu \mathrm{m}$. The concentration of Cur-NPs on plasma, brain, heart, liver, spleen, lung and kidney of C57BL/6 mice (B-H) (means \pm $\mathrm{SD}, \mathrm{n}=4)$.

\section{Cur-NPs attenuated ICH induced behavioral deficits}

In the rotarod test, ICH lead to an obvious drop in the latency to fall, while Cur-NPs caused an obvious increase in latency to fall. Besides, Cur-NPs effectively reversed 
the ICH-induced increase in the number of drops (Fig. 4A). In the pole climbing test, ICH lead to a marked increase in turn downward (T-turn) and the total time (T-total). While Cur-NPs treatment reversed these effects (Fig. 4B). Cut-NPs were significantly more effective than free Cur as a means of mitigating behavioral deficits. Overall, these findings suggested that Cur-NPs can significantly attenuated behavioral deficits in mice.
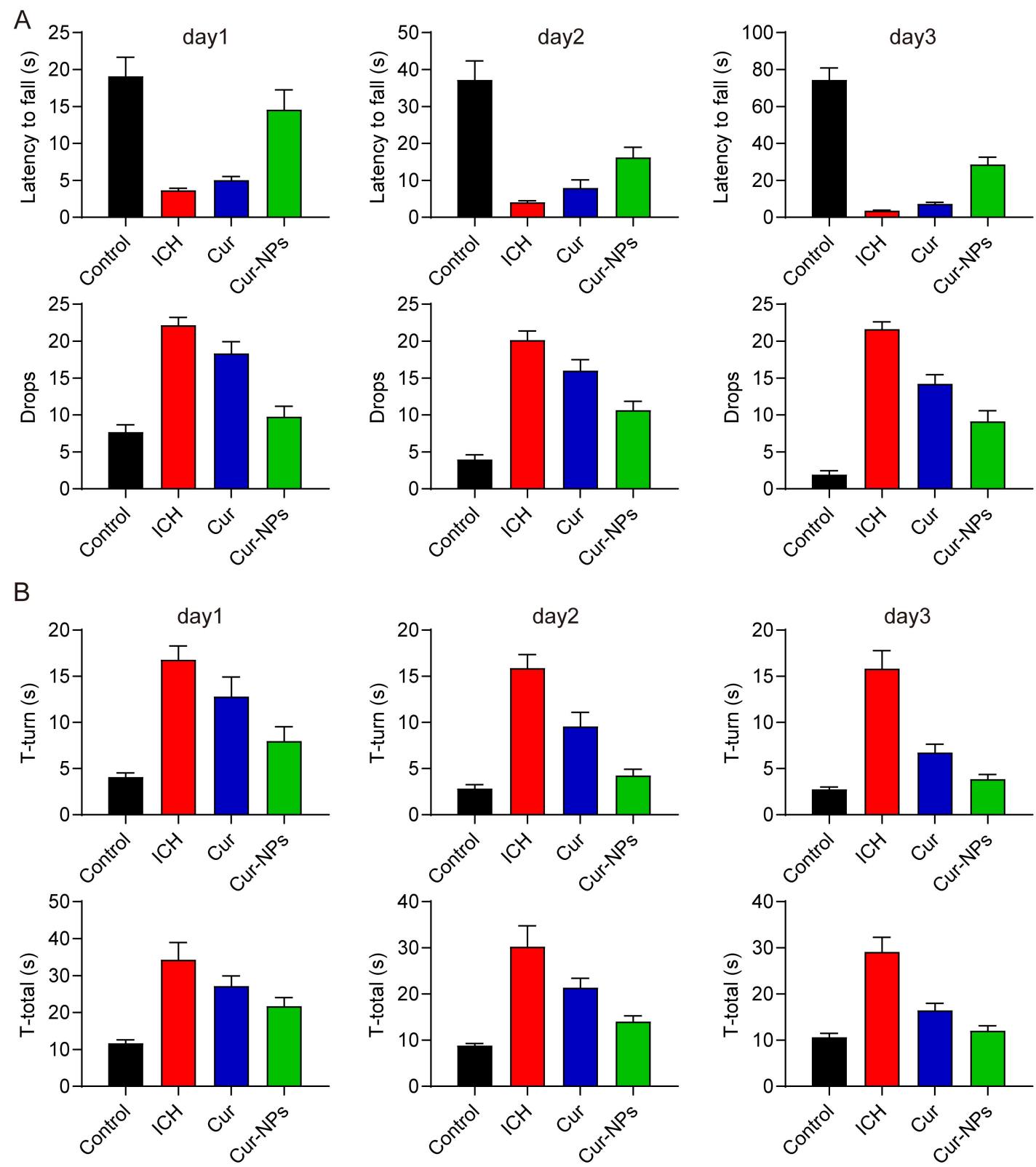
Fig. 4 Effects of Cur-NPs on ICH induced behavioral deficits. A The latency to fall and the number of drops of rotarod tests (means $\pm \mathrm{SD}, \mathrm{n}=12$ ). B The T-turn time and T-total time of pole tests (means $\pm \mathrm{SD}, \mathrm{n}=12$ ).

\section{Cur-NPs decreased the hematoma volumes in the brain of mice}

Cur-NPs treatment obviously decreased the hematoma volumes in the brain of mice compared with the ICH and Cur groups (Fig. 5A, 5B). Moreover, the results of Hematoxylin eosin (HE) staining showed that ICH lead to significant neural loss in the perihematoma brain tissues, while Cur-NPs treatment effectively mitigated the neural damage (Fig. 5C).
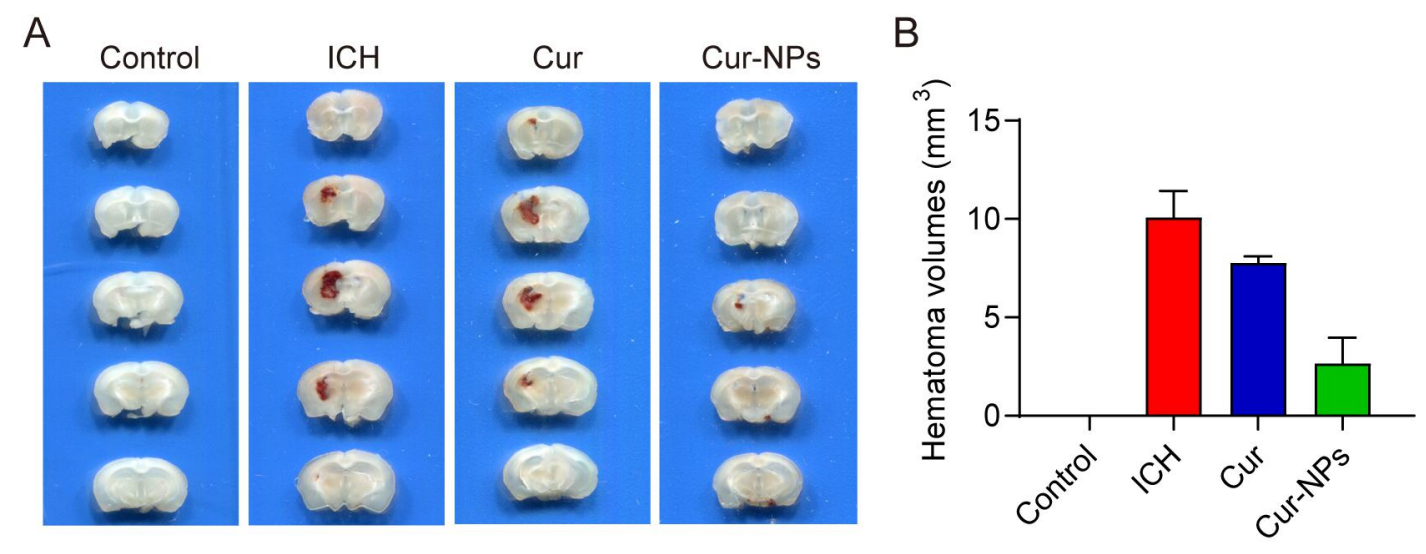

C

$$
\text { Control }
$$

$\mathrm{ICH}$

Cur

Cur-NPs
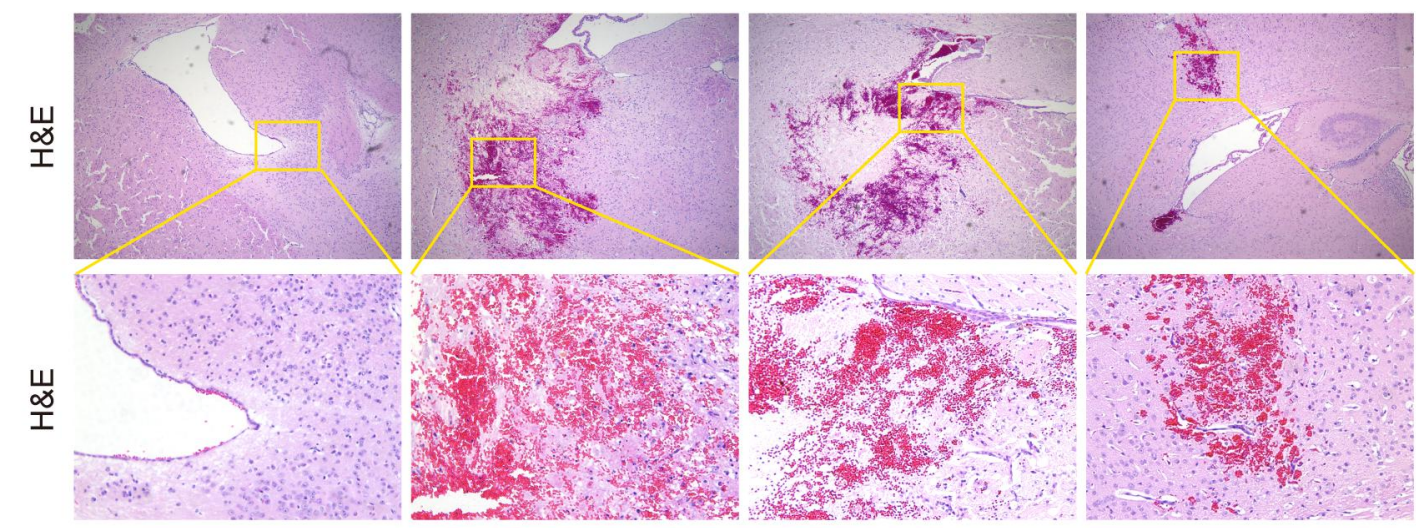
Fig.5 Cur-NPs decreased the hematoma volumes in the brain of mice. A Images of hematoma of ICH mice brain sections. B The hematoma volumes of ICH mice in each group (means $\pm \mathrm{SD}, \mathrm{n}=$ 6). C HE staining of the brain tissues in each group.

\section{Cur-NPs attenuated ICH induced neurological injury}

Nissl staining showed that ICH resulted in obvious neuronal degeneration in the perihematoma brain tissues of ICH mice. In contrast, Cur-NPs significantly attenuated the neuronal degeneration in the perihematoma brain tissues of ICH mice. Cur-NPs exhibited a better curative effect than Cur. Prussian blue staining was conducted to assess the intracellular iron accumulation and distribution in perihematoma brain tissues. The results of Prussian blue staining showed that Cur-NPs markedly decreased the number of Prussian blue positive cells compared with the ICH and Cur groups, suggesting that Cur-NPs was effective to reduce iron deposition in the perihematoma brain tissues. Glutathione peroxidase 4 (GPX4) is a subtype of glutathione peroxidase that can detoxify lipid peroxidase [34]. It is a molecular marker of ferroptosis which can inhibit ferroptosis through decreasing the lipid peroxidization in cells[35, 36]. In this study, it was observed that Cur-NPs administration reduced the compensatory overexpression of GPX4 in the perihematoma region after ICH (Fig. 6). These results thus suggested that Cur-NPs treatment significantly attenuated ferroptosis in ICH. 


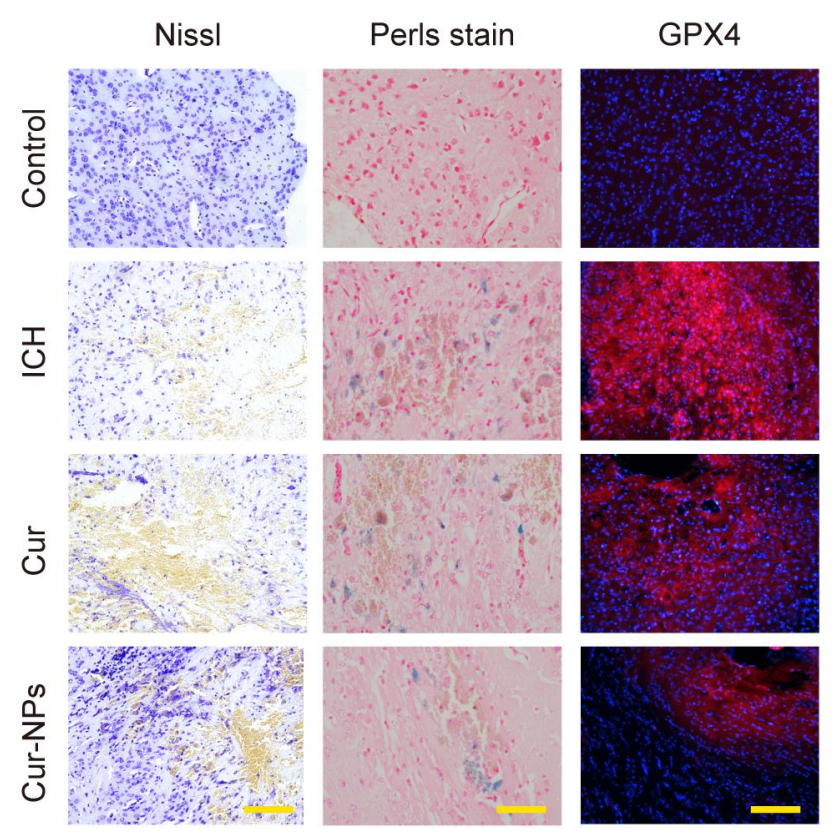

Fig. 6 Representative microphotographs of stained mouse brain sections. Scale bar: $250 \mu \mathrm{m}$.

\section{Cur-NPs inhibited ferroptosis induced by erastin in HT22 cells}

MTT assay showed that conventional-dose Cur-NPs (not higher than $20 \mu \mathrm{M}$ ) existed no obvious toxic effect on the cell viability (Fig.7A). We utilized erastin, a ferroptosis inducer to investigate the anti-ferroptosis effects of Cur-NPs [37]. MTT test showed that Cur-NPs treatment effectively enhanced the cell viability relative to the erastin group and Cur-PM group (Fig.7B). Likewise, the live/dead cell staining assay revealed the consistent results that Cur-NPs dramatically increased the survival rate of HT22 cells (Fig.7C). 

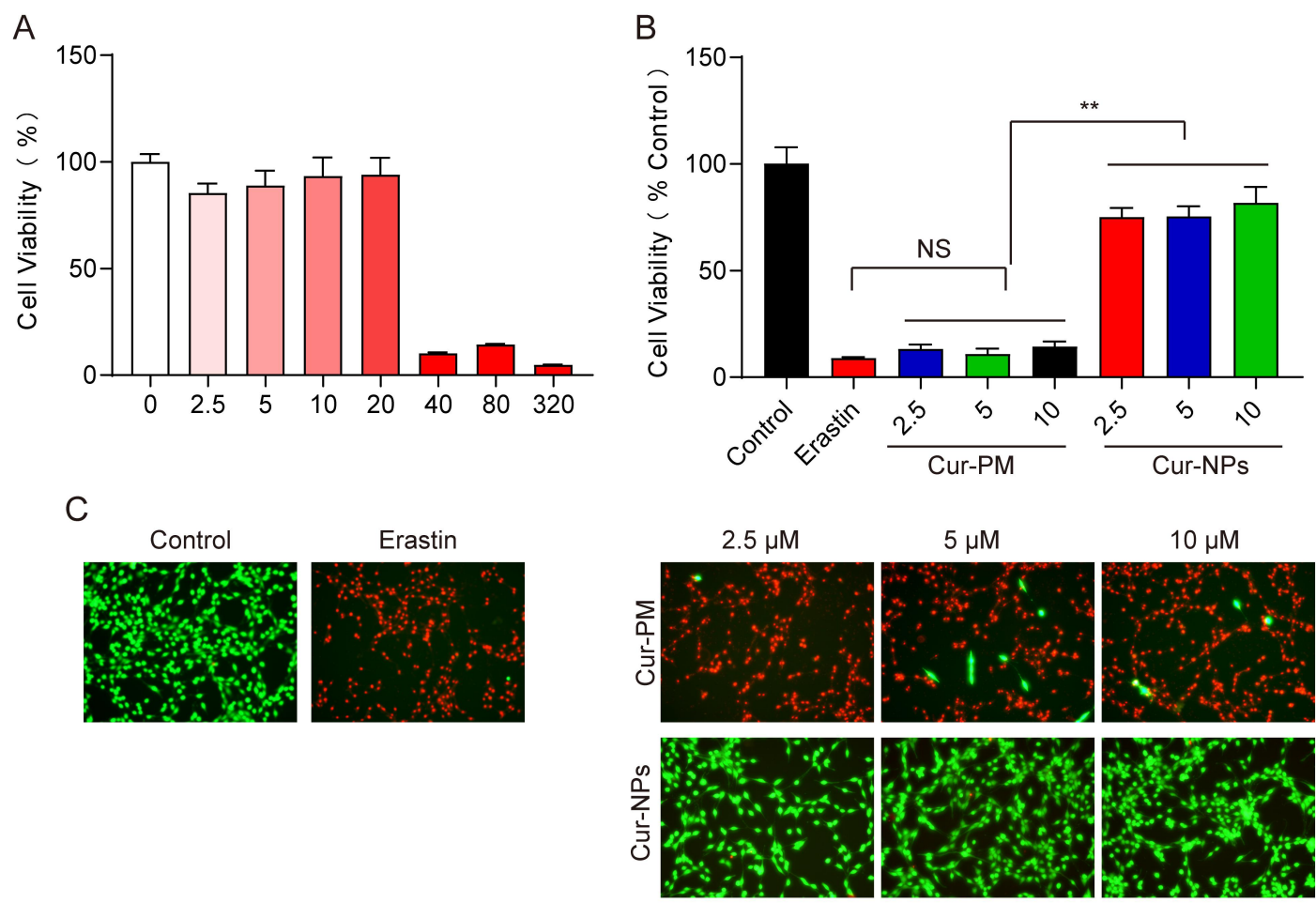

Fig. 7 Cell viability of erastin-induced HT22 cells treated with Cur-NPs and Cur-PM. A MTT assays in response to different concentrations of Cur-NPs. B MTT assays in response to the indicated treatments. C Live/dead staining in response to the indicated treatments.

\section{Conclusions}

In this study, Cur-NPs were made by an anti-solvent precipitation method. Cur-NPs can draw into cells through a number of nonspecific endocytosis mechanisms, mainly mediated via clathrin and plasma membrane microcapsules. Cur-NPs tended to accumulate in the endoplasmic reticulum and lysosome. Moreover, Cur-NPs could transport across physiological barriers and improve Cur accumulation in the plasma and brain. Importantly, Cur-NPs were an effective treatment for ICH through inhibiting ferroptosis. Taken together, Cur-NPs represent a potentially effective strategy to enhance Cur brain delivery and therapeutic efficacy in the treatment of ICH. 


\section{Methods}

\section{Materials}

Cur and PVP k90 was purchased from Nantong Feiyu Biological Technology Co., Ltd. (Nantong, China). mPEG-PTMC (mPEG, MW, 2000Da; PLGA, MW 16000Da) were purchased from the Jinan Daigang Biomaterial Co., Ltd (Jinan, China). 5-(N-ethyl-N-isopropyl)-amiloride (EIPA), LY294002 (LY), CPZ, HS and MßCD were purchased from Sigma Aldrich (MO, USA). C6, Lyso tracker, ER tracker and Mito tracker were obtained from Molecular Probes Inc. (OR, USA). Erastin was purchased from MCE (NJ, USA). MTT solution was obtained from Sigma Aldrich (St. Louis, MO, USA). Live/Death detection kit was obtained from KeyGene Biotech (Nanjing, China). Collagenase Type IV was purchased from the ThermoFisher Scientific (Springfield Township, NJ, USA). Nissl staining kit was purchased from Beyotime Biotechnology Co. Ltd. (shanghai, China). Prussian blue staining kit was procured from Servicebio (Wuhan, China). HE staining kit was obtained from Leagene Biotechnology (Beijing, China). The primary antibody against GPX4 was obtained from Abcam, Inc.(Cambridge, UK). The second antibodies including anti-rabbit IgG (Alexa Fluor 594) were purchased form Cell Signaling Technology (MA, USA).

\section{Cur-NPs preparation and characterization}


In our research, Cur-NPs were made by an anti-solvent precipitation method. An organic phase: Cur $(20 \mathrm{mg} / \mathrm{ml})$ and $\mathrm{mPEG}(2 \mathrm{~K})-\mathrm{PTMC}(16 \mathrm{~K}) 20 \mathrm{mg} / \mathrm{ml}$ were dissolved into acetone. An aqueous phase was prepared through dissolving PVP k90 (2 mg/ml) into water. Next, $0.2 \mathrm{~mL}$ organic phase was poured into $10 \mathrm{~mL}$ aqueous phase, and the mixture was subjected to $1000 \mathrm{rpm} / \mathrm{min}$ stirring to make the Cur-NPs. Additionally, C6 and mPEG-PTMC were mixed in the organic phase at a ratio of 1:30 to make C6-NPs. To remove the residual solvent, the obtained C6-NPs were stirred in the dark at room temperature for $2 \mathrm{~h}$. The physicochemical properties of Cur-NPs were analyzed, including morphology by a TEM, the particle size, zeta potential and crystalline patterns by XRD.

\section{Endocytosis mechanism detection of Cur-NPs}

To analyze the endocytic mechanisms of Cur-NPs, MDCK cells were incubated with five different endocytosisinhibitors including EIPA, LY, CPZ, HS and M $\beta C D$ for 30 $\min [38]$. Next, the C6-NPs were incubated with MDCK cells for $1 \mathrm{~h}$. Then the liquid was removed and the cells were washed three times in PBS. After that, the samples were fixed with $4 \%$ paraformaldehyde (PFA) for $5 \mathrm{~min}$. At last, the images of the samples were obtained by using a confocal laser scanning microscopy (CLSM; TCS SPE II, Leica, Germany).

\section{In vitro cellular uptake of Cur-NPs}

MDCK cells were seeded on coverslips at a cell density of $5 \times 10^{4}$ in 24 -well plates. 
Next, we cultured C6-NPs with complete medium to $0.5 \mu \mathrm{g} / \mathrm{mL}, 1.0 \mu \mathrm{g} / \mathrm{mL}$ and 2.0 $\mu \mathrm{g} / \mathrm{mL}$ respectively. The incubation of medium was removed at different time points $(10,30,60 \mathrm{~min})$. Subsequently, we fixed the samples with $4 \%$ PFA. At last, the images were acquired using a fluorescence microscope (model DMi8, Leica, Germany) at $488 \mathrm{~nm}$. In the present work, we selected Lyso, ER and Mito as detection organelles. The cells were mixed with Lyso tracker, ER tracker and Mito tracker for $120 \min [39]$. Sequentially, the samples were rinsed with serum-free medium for 5 min three times and fixed with C6-NPs. The colocalization of C6-NPs with the organelles were imaged using a confocal laser scanning microscopy (CLSM; TCS SPE II, Leica, Germany).

\section{In vivo biodistribution of Cur-NPs in zebrafish}

In this study, we obtained zebrafish (Danio rerio) from the China Zebrafish Resource Center (Wuhan, China). The zebrafish were housed on a $14 \mathrm{~h} / 10 \mathrm{~h}$ light/dark cycle. Male and female zebrafish were separated at a 1:2 ratio in a $1 \mathrm{~L}$ tank overnight when they were mature. The screen was separated and embryos that had been fertilized were collected after the light cycle. Pigment formation was blocked by 1-phenyl-2thiourea. To determine the biodistribution of Cur-NPs after oral administration, we incubated zebrafish embryos at 7dpf in C6/Cur-NPs solutions with a C6 concentration of $400 \mathrm{ng} / \mathrm{ml}$ for 15 and $60 \mathrm{~min}$. After the treatment, the biodistribution of C6/Cur-NPs were observed and imaged using a fluorescence microscope (DMi8, Leica, Germany). 


\section{Pharmacokinetic analysis of Cur-NPs in mice}

Male C57BL/6 mice ( 8 weeks old) were utilized to carry out the Cur-NPs pharmacokinetics analysis. The animals were housed under a $12 \mathrm{~h}$ day/night cycle at $25{ }^{\circ} \mathrm{C}$ with $55 \%$ relative humidity. They had free access to water and standard diet. Cur-NPs or an equivalent Cur dose $(5 \mathrm{mg} / \mathrm{kg})$ in $1.5 \mathrm{mg} / \mathrm{ml} \mathrm{HPMC} \mathrm{(controls)} \mathrm{was}$ orally administered to mice. Serum samples were collected from the tail veins of each rat $(\mathrm{n}=4$ per time point) at $1,2,4$ and $8 \mathrm{~h}$ after administration. Samples were centrifuged at $3500 \mathrm{rpm}$ for $10 \mathrm{~min}$, and supernatant was removed for analysis. Main organs samples ( $n=4$ per time point) were collected via anesthetizing the mice after treatment at the 1, 2, 4 and $8 \mathrm{~h}$ time points. Then, Cur concentrations in the plasma and organs were detected by LC-MS/MS[40] .

\section{Establishment of ICH mouse model}

Male C57BL/6 mice (8-10 weeks old) were provided by the Experimental Animal Center of Guangzhou University of Chinese Medicine (Guangzhou, China). We established an ICH animal model according to previously discribed as following[41]: The mice were placed in a prone position and the head was stabilized using a stereotaxic frame after anesthetization. We made a $1 \mathrm{~mm}$ burr hole by a dental drill at the position $2.0 \mathrm{~mm}$ lateral right of the bregma and $3.5 \mathrm{~mm}$ deep of the brain of mice. Sequentially, 0.1U type IV collagenase was slowly injected into the hole to cause acute ICH. Meanwhile, control animals were administered with an equal 
volume of saline. Ethical approval for the animal experiments was granted by the Animal Ethics Committee of Guangzhou University of Chinese Medicine, which are consistent with regulations for the care and use of experimental animals in China.

\section{Drug treatment}

The mice were randomly divided into four groups: Control group, ICH group, Cur group and Cur-NPs group (n=6/group). The Cur solution or Cur-NPs solution was administered to the mice in the Cur group and Cur-NPs group respectively by gastric gavage 2 hours after ICH injury onset. To achieve $20 \mathrm{mg} / \mathrm{kg} /$ day dosage, the drug administration was conducted twice daily successively for the 3 days after operation. The remaining two groups received an equal volumes of saline through oral gavage.

\section{Behavioral test}

We assessed the impact of Cur-NPs on motor ability of ICH mice by a rotarod test and a pole climbing test. In the rotarod test, the mice were positioned on a rotarod at a speed of 20 rounds per minute for $120 \mathrm{~s}$. The latency to fall and the number of drops within $120 \mathrm{~s}$ were recorded. In the pole climbing test, the mice were placed on the top of a pole with a diameter of $0.9 \mathrm{~cm}$ and a height of $50 \mathrm{~cm}$, and allowed to climb down at 5-min intervals without interference. The time a mouse required to turn downward (T-turn) and the total time (T-total) a mouse required to reach the bottom were recorded. 


\section{Hematoma assessment}

After the behavioral tests, the animals were sacrificed and the whole brains were carefully removed and cut into $1 \mathrm{~mm}$ thick brain sections after perfusion with PBS. Sequentially, the images were acquired by an Epson Perfection V370 photo scanner (Epson China, Beijing, China). The hematoma volume of each section measured by using ImageJ software. Hematoma volume in cubic millimeters was calculated as the mean of the summation of the hematoma areas multiplied by the interslice distance $(1 \mathrm{~mm})$.

\section{Brain paraffin slice preparation}

The mice were perfused transcardially with PBS followed by $4 \%$ PFA. Next, the animals were decapitated and the whole brains were removed and stored in $4 \%$ PFA. The brains were then paraffin-embedded and cut into $5 \mu \mathrm{m}$ thick sections for the following analysis.

\section{HE staining}

HE staining was conducted to investigate the effect of Cur-NPs on neuronal loss in the perihematoma brain tissues of mice. The paraffin sections were deparaffinized in xylene and rehydrated with different concentrations of alcohol and distilled water. The sections were stained with hematoxylin solution for $5 \mathrm{~min}$ and eosin solution for $1 \mathrm{~min}$. Next, the sections were dehydrated with alcohol, cleared with xylene and 
mounted with neutral gum. The images were acquired using an optical microscope (DMi8, Leica, Germany).

\section{Nissl staining}

Nissl staining was performed to evaluate the impact of Cur-NPs on neuronal degeneration in the perihematoma brain tissues of mice. The paraffin sections were dewaxed in xylene and rehydrated using different concentrations of ethanol. Next, the sections were stained with $1 \%$ toluidine blue for $10 \mathrm{~min}$. After that, the sections were dehydrated with alcohol gradient and xylene and blocked by neutral gum. The neuronal Nissl bodies in the samples were imaged using an optical microscope (DMi8, Leica, Germany).

\section{Prussian blue staining}

Prussian blue staining was conducted to detect iron accumulation in brain tissues around hematoma. Paraffin-embedded sections were dewaxed with xylene and rehydrated with gradient ethanol. Next, the samples were stained with Perls Prussian blue stain for $15 \mathrm{~min}$ and hematoxylin for $30 \mathrm{~s}$. The samples were then subjected to gradient ethanol dehydration, dimethyl benzene transparency and mounting on neutral resin cover slides. Finally, the sections were observed and imaged with an optical microscope (DMi8, Leica, Germany).

\section{Immunofluorescence staining}


The brain $30 \mu \mathrm{m}$ thick frozen tissue sections were saturated and permeabilized in $0.1 \%$ Triton $\mathrm{X}-100$ and blocked with the goat serum at room temperature. Then the samples were incubated with anti-GPX4 at $4{ }^{\circ} \mathrm{C}$ overnight. The sections were then incubated with goat anti-rabbit IgG (Alexa Fluor 594) in the dark for 1 hour. After DAPI nuclear staining, images were captured using a fluorescent microscope (DMI8, Leica, Germany) and the positive cells enumerated from 3-4 different views in each sample.

\section{Cell viability assay}

MTT assay was conducted to determine the effect of Cur-NPs on cell viability. In our present work, different concentrations of drugs were added to HT22 cells treated with erastin for $24 \mathrm{~h}$. Then the samples were inculated with $90 \mu \mathrm{L}$ DMEM and $10 \mu \mathrm{L}$ MTT solution for $4 \mathrm{~h}$. Next, the supernatant was discarded and each well was added with $150 \mu \mathrm{L}$ DMSO. Sequentially, the plate was shaken on an oscillator for $10 \mathrm{~min}$. The cell viability was determined by calculating the absorbance at $490 \mathrm{~nm}$ using a microplate reader (Multiskan FC, Thermo Scientific, United States).

\section{Live / dead cell staining assay}

Live and dead cell staining assay test was performed to analyze the impact of Cur-NPs on cell survival. Erastin-induced HT22 cells were incubated with indicated concentrations of drugs for $24 \mathrm{~h}$. Sequentially, the supernatant was discarded and the samples were stained with staining working solution $(2 \mu \mathrm{M}$ calcein $\mathrm{AM}, 8 \mu \mathrm{M}$ PI) for 
$30 \mathrm{~min}$ in the dark. The images were acquired using a fluorescence microscope (DMi8, Leica, Germany).

\section{Statistical analysis}

Data are expressed as the means \pm standard deviation (SD) and analyzed using one-way analysis of variance (ANOVA) followed by two-tailed Student's $t$ test. The threshold for significance was $P<0.05$.

\section{Authors' contributions}

$\mathrm{CY}, \mathrm{MH}$ and RL performed the preparation and characteristics of the NPs. YY and LZ conducted the animal experiments. LD and SS performed the in vitro experiments. ML helped in the analysis of biological data. QW, TC and YM supervised the whole work. All authors read and approved the final manuscript.

\section{Author details}

${ }^{1}$ Science and Technology Innovation Center, Guangzhou University of Chinese Medicine, Guangzhou 510405, China. ${ }^{2}$ The First Affiliated Hospital of Guangzhou University of Chinese Medicine, Guangzhou 510405, China. ${ }^{3}$ Laboratory Animal Center, Guangzhou University of Chinese Medicine, Guangzhou 510405, China. ${ }^{4}$ Clinical Medical College of Acupuncture Moxibustion and Rehabilitation, Guangzhou University of Chinese Medicine, Guangzhou 510405, China. ${ }^{5}$ The Second 
Affiliated Hospital of Guangzhou University of Chinese Medicine, Guangzhou 510120, China.

\section{Acknowledgements}

This work was supported by Key laboratory project of colleges and universities in Guangdong province (2019KSYS005), the Guangdong province science and technology plan international cooperation project (2020A0505100052), the Guangdong Basic and Applied Basic Research Foundation (2019B1515120043), the key Project of Basic Research of Shenzhen (JCYJ20200109113603854), and the Guangdong Provincial Natural Science Foundation of China (2018A030310623).

\section{Competing interests}

The authors declare that they have no competing interests.

\section{References}

1. Ding, D.; Sekar, P.; Moomaw, C. J.; Comeau, M. E.; James, M. L.; Testai, F.; Flaherty, M. L.; Vashkevich, A.; Worrall, B. B.; Woo, D.; Osborne, J. Venous Thromboembolism in Patients With Spontaneous Intracerebral Hemorrhage: A Multicenter Study. Neurosurgery 2019, 84, E304-304E310.

2. Béjot, Y.; Blanc, C.; Delpont, B.; Thouant, P.; Chazalon, C.; Daumas, A.; Osseby, G. V.; Hervieu-Bègue, M.; Ricolfi, F.; Giroud, M.; Cordonnier, C. Increasing early ambulation disability in spontaneous intracerebral hemorrhage survivors. Neurology 2018, 90, e2017-2017e2024. 
3. Zhou, S. Y.; Cui, G. Z.; Yan, X. L.; Wang, X.; Qu, Y.; Guo, Z. N.; Jin, H. Mechanism of Ferroptosis and Its Relationships With Other Types of Programmed Cell Death: Insights for Potential Interventions After Intracerebral Hemorrhage. Front Neurosci 2020, 14, 589042.

4. Chen, B.; Chen, Z.; Liu, M.; Gao, X.; Cheng, Y.; Wei, Y.; Wu, Z.; Cui, D.; Shang, H. Inhibition of neuronal ferroptosis in the acute phase of intracerebral hemorrhage shows long-term cerebroprotective effects. Brain Res. Bull. 2019, 153, 122-132.

5. Stockwell BR, Friedmann Angeli JP, Bayir H, et al. Ferroptosis: A Regulated Cell Death Nexus Linking Metabolism, Redox Biology, and Disease. Cell. 2017;171(2):273-85.

6. Li Y, Liu Y, Wu P, Tian Y, Liu B, Wang J, Bihl J, Shi H. Inhibition of Ferroptosis Alleviates Early Brain Injury After Subarachnoid Hemorrhage In Vitro and In Vivo via Reduction of Lipid Peroxidation. Cell Mol Neurobiol. 2021;41(2):263-78.

7. Weiland A, Wang Y, Wu W, Lan X, Han X, Li Q, Wang J. Ferroptosis and Its Role in Diverse Brain Diseases. Mol Neurobiol. 2019;56(7):4880-93.

8. Sun, S.; Li, Y.; Zhang, H.; Gao, H.; Zhou, X.; Xu, Y.; Yan, K.; Wang, X. Neuroendoscopic Surgery versus Craniotomy for Supratentorial Hypertensive Intracerebral Hemorrhage: A Systematic Review and Meta-Analysis. World neurosurgery 2020, 134, 477-488.

9. Hemphill, J. C. 3rd; Amin-Hanjani, S. Cerebellar Intracerebral Hemorrhage: Time for Evidence-Based Treatment. JAMA 2019, 322, 1355-1356.

10. Kuramatsu, J. B.; Biffi, A.; Gerner, S. T.; Sembill, J. A.; Sprügel, M. I.; Leasure, A.; Sansing, L.; Matouk, C.; Falcone, G. J.; Endres, M.; Haeusler, K. G.; Sobesky, J.; Schurig, J.; Zweynert, S.; Bauer, M.; Vajkoczy, P.; Ringleb, P. A.; Purrucker, J.; Rizos, T.; Volkmann, J.; Müllges, W.; Kraft, P.; Schubert, A. L.; Erbguth, F.; Nueckel, M.; Schellinger, P. D.; Glahn, J.; Knappe, U. J.; Fink, G. R.; Dohmen, C.; Stetefeld, H.; Fisse, A. 
L.; Minnerup, J.; Hagemann, G.; Rakers, F.; Reichmann, H.; Schneider, H.; Rahmig, J.; Ludolph, A. C.;

Stösser, S.; Neugebauer, H.; Röther, J.; Michels, P.; Schwarz, M.; Reimann, G.; Bäzner, H.; Schwert, H.;

Claßen, J.; Michalski, D.; Grau, A.; Palm, F.; Urbanek, C.; Wöhrle, J. C.; Alshammari, F.; Horn, M.;

Bahner, D.; Witte, O. W.; Günther, A.; Hamann, G. F.; Hagen, M.; Roeder, S. S.; Lücking, H.; Dörfler, A.;

Testai, F. D.; Woo, D.; Schwab, S.; Sheth, K. N.; Huttner, H. B. Association of Surgical Hematoma

Evacuation vs Conservative Treatment With Functional Outcome in Patients With Cerebellar Intracerebral

Hemorrhage. JAMA 2019, 322, 1392-1403.

11. Holste, K.; Xia, F.; Garton, H.; Wan, S.; Hua, Y.; Keep, R. F.; Xi, G. The role of complement in brain injury following intracerebral hemorrhage: A review. Exp. Neurol. 2021, 340, 113654.

12. Feng, C.; Huang, S.; Zhou, X.; Wang, L.; Cui, X.; Chen, L.; Lv, F.; Li, T. Interventional Ultrasound Assisted Early Local Hemostatic Drug Therapy in Traumatic Intracerebral Hemorrhage. Ultrasound Med Biol 2020, $46,180-187$.

13. Deng, S.; Feng, S.; Wang, W.; Zhao, F.; Gong, Y. Biomarker and Drug Target Discovery Using Quantitative Proteomics Post-Intracerebral Hemorrhage Stroke in the Rat Brain. J. Mol. Neurosci. 2018, 66, 639-648.

14. Wang, H. L.; Zeng, H.; Xu, M. B.; Zhou, X. L.; Rong, P. Q.; Jin, T. Y.; Wang, Q.; Zheng, G. Q. Efficacy and Safety of Chinese Herbal Medicine for Primary Intracerebral Hemorrhage: A Systematic Review of Randomized Controlled Trials. Front Pharmacol 2019, 10, 1139.

15. Dong, W.; Yang, B.; Wang, L.; Li, B.; Guo, X.; Zhang, M.; Jiang, Z.; Fu, J.; Pi, J.; Guan, D.; Zhao, R. Curcumin plays neuroprotective roles against traumatic brain injury partly via Nrf2 signaling. Toxicol. Appl. Pharmacol. 2018, 346, 28-36.

16. Abu-Taweel, G. M. Neurobehavioral protective properties of curcumin against the mercury chloride treated mice offspring. Saudi journal of biological sciences 2019, 26, 736-743. 
17. Zhang, J.; Tang, L.; Li, G. S.; Wang, J. The anti-inflammatory effects of curcumin on renal ischemia-reperfusion injury in rats. Ren Fail 2018, 40, 680-686.

18. Wang, B. F.; Cui, Z. W.; Zhong, Z. H.; Sun, Y. H.; Sun, Q. F.; Yang, G. Y.; Bian, L. G. Curcumin attenuates brain edema in mice with intracerebral hemorrhage through inhibition of AQP4 and AQP9 expression. Acta Pharmacol. Sin. 2015, 36, 939-948.

19. de Souza Ferreira, S. B.; Bruschi, M. L. Improving the bioavailability of curcumin: is micro/nanoencapsulation the key. Ther Deliv 2019, 10, 83-86.

20. Tai, K.; Rappolt, M.; Mao, L.; Gao, Y.; Yuan, F. Stability and release performance of curcumin-loaded liposomes with varying content of hydrogenated phospholipids. Food Chem 2020, 326, 126973.

21. Muddineti, O. S.; Kumari, P.; Ghosh, B.; Biswas, S. Transferrin-Modified Vitamin-E/Lipid Based Polymeric Micelles for Improved Tumor Targeting and Anticancer Effect of Curcumin. Pharm. Res. 2018, 35, 97.

22. Gao, Y.; Ding, S.; Huang, X.; Fan, Z.; Sun, J.; Hai, Y.; Li, K. Development and evaluation of hollow mesoporous silica microspheres bearing on enhanced oral delivery of curcumin. Drug Dev Ind Pharm 2019, $45,273-281$

23. Ten-Doménech, I.; Martínez-Pérez-Cejuela, H.; Simó-Alfonso, E. F.; Torres-Cartas, S.; Meseguer-Lloret, S.; Herrero-Martínez, J. M. Polymer-based materials modified with magnetite nanoparticles for enrichment of phospholipids. Talanta 2018, 180, 162-167.

24. Park, K.; Skidmore, S.; Hadar, J.; Garner, J.; Park, H.; Otte, A.; Soh, B. K.; Yoon, G.; Yu, D.; Yun, Y.; Lee, B. K.; Jiang, X.; Wang, Y. Injectable, long-acting PLGA formulations: Analyzing PLGA and understanding microparticle formation. J Control Release 2019, 304, 125-134.

25. Asai, M.; Zhao, D.; Kumar, S. K. Accurate estimation of the polymer coverage of hairy nanoparticles. Soft Matter 2018, 14, 7906-7915. 
26. Teresi, R.; Marullo, S.; Gambarotti, C.; Parisi, F.; Megna, B.; Lazzara, G.; D\&\#39; Anna, F.; Dintcheva, N. T. Improvement of oxidation resistance of polymer-based nanocomposites through sonication of carbonaceous nanoparticles. Ultrason Sonochem 2020, 61, 104807.

27. Hua Z, Jones JR, Thomas M, Arno MC, Souslov A, Wilks TR, O\&\#39, Reilly RK. Anisotropic polymer nanoparticles with controlled dimensions from the morphological transformation of isotropic seeds. Nat Commun. 2019;10(1):5406.

28. Cheng, K. K.; Yeung, C. F.; Ho, S. W.; Chow, S. F.; Chow, A. H.; Baum, L. Highly stabilized curcumin nanoparticles tested in an in vitro blood-brain barrier model and in Alzheimer's disease $\operatorname{Tg} 2576$ mice. AAPS J 2013, 15, 324-336.

29. Wang, Y.; Song, J.; Chow, S. F.; Chow, A. H.; Zheng, Y. Particle size tailoring of ursolic acid nanosuspensions for improved anticancer activity by controlled antisolvent precipitation. Int J Pharm 2015, 494, 479-489.

30. He, B.; Jia, Z.; Du, W.; Yu, C.; Fan, Y.; Dai, W.; Yuan, L.; Zhang, H.; Wang, X.; Wang, J.; Zhang, X.; Zhang, Q. The transport pathways of polymer nanoparticles in MDCK epithelial cells. Biomaterials 2013, 34, $4309-4326$

31. Fako, V. E.; Furgeson, D. Y. Zebrafish as a correlative and predictive model for assessing biomaterial nanotoxicity. Adv. Drug Deliv. Rev. 2009, 61, 478-486.

32. Hauser, A. K.; Mitov, M. I.; Daley, E. F.; McGarry, R. C.; Anderson, K. W.; Hilt, J. Z. Targeted iron oxide nanoparticles for the enhancement of radiation therapy. Biomaterials 2016, 105, 127-135.

33. Wang HY, Cao PP, He ZY, He XW, Li WY, Li YH, Zhang YK. Targeted imaging and targeted therapy of breast cancer cells via fluorescent double template-imprinted polymer coated silicon nanoparticles by an epitope approach. Nanoscale. 2019;11(36):17018-30. 
34. Zhang, Z.; Wu, Y.; Yuan, S.; Zhang, P.; Zhang, J.; Li, H.; Li, X.; Shen, H.; Wang, Z.; Chen, G. Glutathione peroxidase 4 participates in secondary brain injury through mediating ferroptosis in a rat model of intracerebral hemorrhage. Brain Res. 2018, 1701, 112-125.

35. Imai, H.; Matsuoka, M.; Kumagai, T.; Sakamoto, T.; Koumura, T. Lipid Peroxidation-Dependent Cell Death Regulated by GPx4 and Ferroptosis. Curr. Top. Microbiol. Immunol. 2017, 403, 143-170.

36. Stockwell BR. A powerful cell-protection system prevents cell death by ferroptosis. Nature. 2019;575(7784):597-8.

37. Yang Y, Luo M, Zhang K, et al. Nedd4 ubiquitylates VDAC2/3 to suppress erastin-induced ferroptosis in melanoma. Nat Commun. 2020;11(1):433.

38. Yu C, He B, Xiong MH, et al. The effect of hydrophilic and hydrophobic structure of amphiphilic polymeric micelles on their transport in epithelial MDCK cells. Biomaterials. 2013;34(26):6284-98.

39. Chen, Z.; Ma, T.; Huang, C.; Zhang, L.; Zhong, J.; Han, J.; Hu, T.; Li, J. Efficiency of transcellular transport and efflux of flavonoids with different glycosidic units from flavonoids of Litsea coreana L. in a MDCK epithelial cell monolayer model. Eur J Pharm Sci 2014, 53, 69-76.

40. Yang KY, Lin LC, Tseng TY, Wang SC, Tsai TH. Oral bioavailability of curcumin in rat and the herbal analysis from Curcuma longa by LC-MS/MS. J Chromatogr B Analyt Technol Biomed Life Sci. $2007 ; 853(1-2): 183-9$

41. Mo, Y.; Duan, L.; Yang, Y.; Liu, W.; Zhang, Y.; Zhou, L.; Su, S.; Lo, P. C.; Cai, J.; Gao, L.; Liu, Q.; Chen, X.; Yang, C.; Wang, Q.; Chen, T. Nanoparticles improved resveratrol brain delivery and its therapeutic efficacy against intracerebral hemorrhage. Nanoscale 2021, 13, 3827-3840. 